\title{
Survey of Hand and Upper Extremity Injuries Among Rock Climbers
}

HAND

2017, Vol. 12(4) 389-394

(c) The Author(s) 2016

Reprints and permissions:

sagepub.com/journalsPermissions.nav

DOI: $10.1177 / 1558944716679600$

hand.sagepub.com

\author{
Clayton E. Nelson', Ghazi M. Rayan², Dustin I. Judd', Kai Ding', \\ and Julie A. Stoner
}

\begin{abstract}
Background: Rock climbing first evolved as a sport in the late 18th century. With its growing popularity, the number of rock climbing-related injuries has potential to increase, spurring a rise in the number of articles associated with it. Despite the available literature, there remains a paucity of information about upper extremity injuries sustained by rock climbers, and no studies to date have focused on gender-specific injuries. Methods: A 24-question online survey was distributed to rock climbers about upper extremity injuries sustained during rock climbing. Statistical analysis was used to study association between participants' demographics and injuries. Results: A total of 397 participants responded to the survey. Mean age was 32.5 years with males comprising $85 \%$. No significant differences in demographics or climbing behaviors were found between males and females. Ninety percent of participants reported sustaining an upper extremity injury. Fingers were the most common injury followed by shoulder/arm and elbow/forearm. Our study found females to be more likely to report a rock climbing-related injury, and more likely to undergo surgery for it. Conclusion: Female rock climbers were significantly more likely to report a shoulder/upper arm injury and were also more likely to report undergoing surgery compared with males, where these differences were not due to age or climbing behaviors. Further investigation is warranted into the association between shoulder injuries and female athletes to determine how the gender differences relate to extent of injury as well as health service utilization behaviors.
\end{abstract}

Keywords: rock climbing, upper extremity injuries, hand injuries, rock climbers, gender specific injuries

\section{Introduction}

Rock climbing first evolved as a sport in the late 18th and early 19th century as a Swiss and British activity., ${ }^{4,10}$ In 1989 , it was estimated that approximately 100000 people scaled rocks in the United States, whereas a 2006 study estimated approximately 9 million people were participating in rock climbing. ${ }^{1,411,13}$ Furthermore, with the construction of readily available artificial rock climbing walls, rock climbing has evolved from a sport of the elite to an activity involving wide ranges and levels of experience. ${ }^{7,11,15}$ With its growing popularity, the number of rock climbing-related injuries has potential to increase which has spurred a rise in the number of published articles associated with rock climbing. ${ }^{7}$ A recent National Center for Biotechnology Information literature review article published by Pegoli et al found 143 original articles published from 2004 to 2014 related to rock climbing; 30 of these articles are directly related to injuries among rock climbers. ${ }^{14}$ Despite the available literature, there remains a paucity of information about upper extremity injuries sustained by rock climbers, and no studies to date have focused on gender-specific injuries in rock climbers. The purpose of our survey was to evaluate upper extremity injuries among rock climbers; specifically examining differences in climbing practices, experience levels, and postinjury treatment between male and female rock climbers.

\section{Materials and Methods}

A 24-question survey (see Online Appendix) was designed to obtain information concerning upper extremity injuries among rock climbers. The questionnaire was uploaded to the SurveyMonkey website where a link was created for distribution. The link was then emailed to members of local climbing clubs and gyms within the state of Oklahoma. The link to the survey was ultimately posted on the Internet

\footnotetext{
'University of Oklahoma, Oklahoma City, USA

${ }^{2}$ INTEGRIS Baptist Medical Center, Oklahoma City, OK, USA

Supplemental material is available in the online version of the article.

Corresponding Author:

Ghazi M. Rayan, Hand Surgery Division, INTEGRIS Baptist Medical Center, 3366 NW Expressway, Ste. 700, Oklahoma City, OK 73II2, USA.

Email: lora.nall@integrisok.com
} 
Table I. Comparison of Email and Forum Respondents.

\begin{tabular}{|c|c|c|c|}
\hline Variable & Email $(n=76)$ & Forum $(n=321)$ & $P$ value \\
\hline Age, $y^{a}$ & $33.79(11.45)$ & $32.25(11.08)$ & .28 \\
\hline Gender & & & .54 \\
\hline Female & $13(17 \%)$ & $46(14 \%)$ & \\
\hline Male & $63(83 \%)$ & $275(86 \%)$ & \\
\hline \multicolumn{4}{|l|}{ Climbing type } \\
\hline Bouldering & $59(78 \%)$ & $242(75 \%)$ & .68 \\
\hline Top roping & $54(71 \%)$ & $255(79 \%)$ & .11 \\
\hline Lead & $66(87 \%)$ & $296(92 \%)$ & .14 \\
\hline Crack & $54(71 \%)$ & $221(69 \%)$ & .71 \\
\hline Other (ice, indoor wall, etc.) & $45(59 \%)$ & $184(57 \%)$ & .76 \\
\hline Training level & & & .59 \\
\hline Beginner or leisure climbers & $57(75 \%)$ & $222(69 \%)$ & \\
\hline Amateur & $15(20 \%)$ & $81(25 \%)$ & \\
\hline Professional & $4(5 \%)$ & $18(6 \%)$ & \\
\hline Years of climbing & & & .072 \\
\hline$<1$ & $7(9 \%)$ & $9(3 \%)$ & \\
\hline $\mathrm{I}-2$ & $10(13 \%)$ & $49(15 \%)$ & \\
\hline $3-4$ & $10(13 \%)$ & $60(19 \%)$ & \\
\hline $5-6$ & $5(7 \%)$ & $40(12 \%)$ & \\
\hline $7-8$ & $4(5 \%)$ & $18(6 \%)$ & \\
\hline$>8$ & $40(53 \%)$ & 145 (45\%) & \\
\hline Climbing frequency & & & $>.90$ \\
\hline Once a year or once every 3 mo & $2(3 \%)$ & $9(3 \%)$ & \\
\hline Once a month & $7(9 \%)$ & $29(9 \%)$ & \\
\hline Once a week & $67(88 \%)$ & $283(88 \%)$ & \\
\hline Grade & & & .50 \\
\hline $5.0-5.8$ & $7(9 \%)$ & $24(7 \%)$ & \\
\hline $5.9-5.12$ & $60(79 \%)$ & $242(75 \%)$ & \\
\hline$>5.12$ & $9(12 \%)$ & $55(17 \%)$ & \\
\hline Train for climbing & & & .40 \\
\hline Yes & $48(63 \%)$ & $219(68 \%)$ & \\
\hline No & $28(37 \%)$ & $102(32 \%)$ & \\
\hline Hands protection & & & .095 \\
\hline Never & $20(26 \%)$ & 117 (36\%) & \\
\hline Sometimes or always & $56(74 \%)$ & $204(64 \%)$ & \\
\hline
\end{tabular}

${ }^{\text {aD }}$ ata presented as mean (standard deviation).

through seven different rock-climbing forums. There were no exclusion criteria. Data were summarized using mean (standard deviation [SD]) or count (percentage). The Cochran-Armitage test was used to assess the trend among ordinal groups. Logistic regression analysis was used to study association among participant demographic characteristics, climbing behaviors, and the nature of injuries. A $P$ value less than .05 was considered to be statistically significant. SAS software version 9.3 was used for data analyses. This study collected only deidentified data using an online survey instrument, without direct interaction between the investigators and respondents, and was therefore not considered to involve human research participants.

\section{Results}

A total number of 397 participants responded to the survey. Seventy-six (19\%) were email participants and $321(81 \%)$ were forum participants. There were no significant differences between the two groups, and therefore we pooled email respondents and forum respondents for analysis (Table 1). The mean age was 32.5 years (range, $14-70$ years). Males comprised $85 \%(\mathrm{n}=338)$ of respondents while females comprised $15 \%(n=59)$. There were numerous forms of rock climbing represented among the respondents with $362(91 \%)$ participating in lead climbing. Most respondents reported climbing for exercise or enjoyment with $70 \%$ 
characterizing themselves as leisure climbers, $24 \%$ as amateur climbers (competing locally) and $6 \%$ as professionals (climbing nationally). Furthermore, $47 \%$ of respondents reported having climbed for more than 8 years.

A total number of 350 (88\%) participants reported climbing at least once per week. We assessed the associations between demographic covariates and the outcome of whether or not any upper extremity injury was sustained. The demographic covariates considered include age, gender, level of experience, years of climbing, frequency of climbing, grade of climbing, training for climbing, and use of protective gear. The only covariate associated with the outcome was the frequency of climbing with the odds of sustaining an upper extremity injury increased as the frequency of climbing increased $(P=.038)$.

A total number of $357(90 \%)$ participants reported sustaining an upper extremity injury. Fingers (41\%) were the most commonly injured, followed by the shoulder/arm $(20 \%)$ and the elbow/forearm (19\%). The most commonly reported injuries were abrasion/bruises $(66 \%)$, followed by lacerations (57\%) and tendon injuries (56\%). Of those reporting a tendon injury, $86 \%$ were reported as a flexor tendon or pulley injury of the hand. Fractures were reported only $8 \%$ of the time. Of the 357 climbers with upper extremity injuries, $56 \%$ reported chronic or residual symptoms with bone and joint pain (ligament instability) being the most common complaint (69\%).

We investigated the association between demographic variables and specific anatomical sites of injury. Looking at hand and finger injuries and treating those who climb at most once every month as the reference group, the odds ratio (OR) of sustaining finger injuries among those who climb once a week was estimated to be 2.49 (95\% confidence interval [CI], 1.27-4.90).

Treating professional climbers as the reference group, the OR of sustaining a finger injury for beginners was 0.19 (95\% CI, 0.01-4.64), leisure climbers 0.18 (95\% CI, 0.040.78 ), and amateur climbers 0.26 (95\% CI, 0.06-1.20). Thus, as the level of experience increases, the odds of finger injury also increase.

We found no significant differences in demographics or climbing behaviors between males and females (Table 2). There were statistical differences in upper extremity injuries sustained between males and females. Females were significantly more likely to report a shoulder/upper arm injury (64\%) compared with males $(45 \%)(P=.018$; Table $2)$. Males and females did not differ in terms of injuries at any other upper extremity location. After adjusting for climbing frequency and years of climbing, the odds of sustaining a shoulder/upper arm injury for females were twice the odds for males (OR, 2.05; 95\% CI, 1.11-3.77, $P=.02$ ).

Among the 357 respondents who sustained an injury to their upper extremity in the past 12 months, females were significantly more likely to report undergoing surgery compared with males $(21 \%$ vs $11 \%, P=.036$; Table 2$)$. Specifically focusing on upper extremity locations among females who sustained a shoulder/upper arm injury, 33\% underwent surgery compared with $16 \%$ of the males who reported a shoulder/upper arm injury $(P=.033)$.

\section{Discussion}

Rock climbing has gained popularity in the last few decades with a wide range and level of experience among participants. ${ }^{7,11}$ With an increasing number of participants, there is potential for increased rock climbing-related injuries. ${ }^{7,15}$ As medical practitioners are treating more of these injuries, there has been a rise in the number of published articles associated with rock climbing injuries. In a recent literature review on rock climbing-related articles, Pegoli et al found 30 articles that discussed injuries among rock climbers. Of these 30 articles, 10 studied upper extremity injuries specifically. ${ }^{14}$ The authors found a higher incidence of injuries in semi and professional athletes, and these were positively related to the amount of hours spent in training. This correlates with Jones et al, who concluded that dedicated climbers participating in different forms of rock climbing frequently and at a higher level of technical difficulty may be more prone to injury. ${ }^{6}$ Our study, finding that the odds of sustaining an upper extremity injury increased as the frequency of climbing increased $(P=.038)$, supports these previous results. This was particularly true for injuries of the shoulder/upper arm, elbow/forearm, and finger $(P=.03)$.

In a survey of 46 climbers, Shea et al found three-fourths of the climbers reported a climbing-related injury; of these injured climbers, almost one-half reported a hand or wrist injury. ${ }^{17}$ Our survey also found tendon injuries (56\%) to be a common injury among climbers, with $86 \%$ of these being flexor tendon or pulley injuries. This too is consistent with previously published data. ${ }^{6-8,15}$ Rooks et al found that the single most common site of injury in climbers was the proximal interphalangeal (PIP) joint due to flexor tendon-type injuries. They documented PIP joint injuries to be present in more than $50 \%$ of elite sport climbers, accounting for nearly $30 \%$ of all injuries. ${ }^{15}$ Similarly, Logan et al reported that flexor tendon injuries were the most common injury sustained by climbers accounting for nearly $25 \%$ of all injuries. ${ }^{8}$ Finally, in Pegoli's literature review, the authors found lesions of the pulley mechanism to be the most common injury among climbers with a $44.3 \%$ reported rate. ${ }^{14}$ The incidence of flexor pulley lesions in rock climbers has been attributed to the high mechanical weight the pulleys bear with excess or prolonged force from the flexor digitorum superficialis (FDS) and flexor digitorum profundus (FDP) tendons. ${ }^{7,8,14,15}$ Cadaveric studies have reproduced these findings, demonstrating partial/complete tears of FDS or FDP, partial/complete tears of A2 or A3, as well as PIP joint subluxation and PIP joint collateral ligaments sprains with prolonged force. ${ }^{9,18}$ 
Table 2. Comparison of Male and Female Respondents.

\begin{tabular}{|c|c|c|c|}
\hline Variable & Female $(n=52)$ & Male $(n=305)$ & $P$ value \\
\hline Age, $y^{a}$ & $30.6(8.7)$ & $32.6(11.0)$ & .15 \\
\hline \multicolumn{4}{|l|}{ Climbing type } \\
\hline Bouldering & $40(77 \%)$ & 235 (77\%) & $>.90$ \\
\hline Top roping & $44(85 \%)$ & $235(77 \%)$ & .22 \\
\hline Lead & $48(92 \%)$ & $279(9 \mid \%)$ & $>.90$ \\
\hline Crack & $33(63 \%)$ & $215(70 \%)$ & .31 \\
\hline Other (ice, indoor wall, etc.) & $26(50 \%)$ & $177(58 \%)$ & .28 \\
\hline Training level & & & .68 (trend test) \\
\hline Beginner or leisure climbers & $36(69 \%)$ & $212(70 \%)$ & \\
\hline Amateur & $16(31 \%)$ & $72(24 \%)$ & \\
\hline Professional & $0(0 \%)$ & $21(7 \%)$ & \\
\hline Years of climbing & & & .16 (trend test) \\
\hline$<1$ & $4(8 \%)$ & $10(3 \%)$ & \\
\hline $\mathrm{I}-2$ & $8(15 \%)$ & $47(15 \%)$ & \\
\hline $3-4$ & $9(17 \%)$ & $52(17 \%)$ & \\
\hline $5-6$ & $9(17 \%)$ & $30(10 \%)$ & \\
\hline $7-8$ & $2(4 \%)$ & $18(6 \%)$ & \\
\hline$>8$ & $20(38 \%)$ & $148(49 \%)$ & \\
\hline Climbing frequency & & & .28 (trend test) \\
\hline Once a year or once every 3 mo & $0(0 \%)$ & $7(2 \%)$ & \\
\hline Once a month & $4(8 \%)$ & $27(9 \%)$ & \\
\hline Once a week & $48(92 \%)$ & $27 \mid(89 \%)$ & \\
\hline Grade & & & .14 (trend test) \\
\hline $5.0-5.8$ & $4(8 \%)$ & $22(7 \%)$ & \\
\hline $5.9-5.12$ & $46(88 \%)$ & $229(75 \%)$ & \\
\hline$>5.12$ & $2(4 \%)$ & $54(18 \%)$ & \\
\hline Train for climbing & & & $>.90$ \\
\hline Yes & $35(67 \%)$ & $206(68 \%)$ & \\
\hline No & $17(33 \%)$ & 99 (32\%) & \\
\hline \multicolumn{4}{|l|}{ Injury location } \\
\hline Shoulder/upper arm & $30(64 \%)$ & $124(45 \%)$ & .018 \\
\hline Elbow/forearm & $20(43 \%)$ & $121(44 \%)$ & .84 \\
\hline Wrist & $9(19 \%)$ & $60(22 \%)$ & .68 \\
\hline Hand & $6(13 \%)$ & $66(24 \%)$ & .086 \\
\hline Fingers & $29(62 \%)$ & 191 (70\%) & .26 \\
\hline Total number of surgeries had & & & .036 \\
\hline 0 (none required) & $4 I(79 \%)$ & 272 (89\%) & \\
\hline $1-3$ & II (2I\%) & $32(10 \%)$ & \\
\hline $4-6$ & $0(0 \%)$ & $\mathrm{I}(<1 \%)$ & \\
\hline Hands protection & & & .075 \\
\hline Never & $12(23 \%)$ & $109(36 \%)$ & \\
\hline Sometimes or always & $40(77 \%)$ & $196(64 \%)$ & \\
\hline
\end{tabular}

${ }^{a}$ Data presented as mean (standard deviation)

Finally, to our knowledge, there have been no studies to date focusing on gender-specific injuries in rock climbers. Our study found females to be significantly more likely to report a shoulder/upper arm injury (64\%) compared with males $(45 \%)(P=.018)$. Males and females did not differ in terms of injuries at any other upper extremity location. After adjusting for climbing frequency and years of climbing, the odds of sustaining a shoulder/ upper arm injury for females were twice those for males (OR, 2.05, $P=.02$ ). Females were also significantly more likely to report having surgery $(21 \%)$ compared with males $(11 \%)$ for their upper extremity injuries. Specifically among the 30 females who sustained a shoulder/upper arm injury, $33 \%$ underwent surgery compared with $16 \%$ 
of males who reported a shoulder/upper arm injury $(P=$ $.033)$. Given that injury and surgery histories were captured in separate questions, we cannot necessarily say that the surgery was performed due to shoulder/upper arm injury; we only know that a given patient had a history of both a surgery and shoulder/upper arm injury. This information raises the concern of whether women are having more shoulder/upper arm injuries because they are competing in different styles of climbing, competing at higher levels, or climbing more frequently than men? When looking at these gender variables, we found no difference in demographic or climbing behaviors between males and females.

There is very limited literature available regarding shoulder/upper arm injuries within the female athlete. Sallis et al reviewed 3767 college athletes comparing injury patterns between men and women in seven sports (basketball, cross country running, soccer, swimming, tennis, and track and water polo) at the intercollegiate level. The authors found a statistically significant gender difference with higher injury incidence in females compared with males for two sports: swimming and water polo $(P<.001)$. Specifically, female swimmers reported a higher rate of shoulder injuries compared with males. ${ }^{16}$ Similarly, Harber in her review, "The Female Athlete Prospective," states that $53 \%$ of adolescent female tennis players reported associated shoulder injuries compared with only $29 \%$ in males. ${ }^{5}$ Finally, Diesselhorst et al found females who participated in martial arts were statistically more likely to sustain injuries to their shoulder compared with males $(P=.049){ }^{3}$ This increased prevalence of shoulder/upper arm injuries among female athletes has been suggested to be associated with laxity within the supporting connective tissue surrounding the shoulder and weakness within the periscapular muscles and rotator cuff resulting in instability and increasing the risk of injury. ${ }^{12}$ In a study evaluating 51 asymptomatic physically active men $(n=24)$ and women $(n=27)$, women demonstrated significantly more anterior glenohumeral joint laxity $(P=.001)$, less anterior joint stiffness $(P=$ $.01)$, and more joint hypermobility $(P=.01)$ than men. ${ }^{2}$ However, there is a lack of data addressing mechanisms of injury and we need a better understanding of the causes of these gender differences. This information could perhaps prompt the development of prevention programs and sport specific periscapular strengthening programs for female climbers.

Finally, our study has limitations. Given the survey was posted on an online forum without inviting specific participants, we are not able to estimate the response rate. As with any survey, the potential for nonresponse bias exists, thus observed associations may not be representative of all rock climbers. The overall number of female participants is somewhat small, and the power to detect male versus female differences in demographic or climbing behaviors is low for some outcomes.

\section{Ethical Approval}

This study was approved by our institutional review board.

\section{Statement of Human and Animal Rights}

All procedures followed were in accordance with the ethical standards of the responsible committee on human experimentation (institutional and national) and with the Helsinki Declaration of 1975, as revised in 2008 .

\section{Statement of Informed Consent}

Informed consent was obtained from all individual participants included in the study.

\section{Declaration of Conflicting Interests}

The author(s) declared no potential conflicts of interest with respect to the research, authorship, and/or publication of this article.

\section{Funding}

The author(s) received no financial support for the research, authorship, and/or publication of this article.

\section{References}

1. Addiss DG, Baker SP. Mountaineering and rock-climbing injuries in US national parks. Ann Emerg Med. 1989;18: 975-979. doi:10.1016/S0196-0644(89)80463-9.

2. Borsa PA, Sauers EL, Herling DE. Patterns of glenohumeral joint laxity and stiffness in healthy men and women. Med Sci Sports Exerc. 2000;32:1685-1690. doi:10.1097/00005768200010000-00004.

3. Diesselhorst M, Rayan G, Pasque C. Survey of upper extremity injuries among martial arts participants. Hand Surg. 2013;18:151-157. doi:10.1142/S0218810413500172.

4. Haas JC, Meyers MC. Rock climbing injuries. Sports Med. 1995;20(3):199-205. doi:10.2165/00007256-199520030-00006.

5. Harber V. The female athlete perspective. Edmonton, $\mathrm{AB}$, Canada: University of Alberta, 2013.

6. Jones G, Asghar A, Llewellyn DJ. The epidemiology of rock-climbing injuries. Br J Sports Med. 2008;42:773-778. doi:10.1136/bjsm.2007.037978.

7. Kubiak EN, Klugman JA, Bosco JA. Hand injuries in rock climbers. Bull NYU Hosp Jt Dis. 2006;64:172-177.

8. Logan AJ, Makwana N, Mason G, Dias J. Acute hand and wrist injuries in experienced rock climbers. Br J Sports Med. 2004;38:545-548.

9. Marco RA, Sharkey NA, Smith TS, Zissimos AG. Pathomechanics of closed rupture of the flexor tendon pulleys in rock climbers. J Bone Joint Surg Am. 1998;80(7): 1012-1019. 
10. Mountain Climbing. The 1996 Grolier Multimedia Encyclopedia on CD-ROM. Danbury, CT: Grolier Electronic Publishing Inc; 1996.

11. Nelson NG, McKenzie LB. Rock climbing injuries treated in emergency departments in the U.S., 1990-2007. Am J Prev Med. 2009;37(3):195-200.

12. Niederbracht Y, Shim AL, Sloniger MA, PaternostroBayles M, Short TH. Effects of a shoulder injury prevention strength training program on eccentric external rotator muscle strength and glenohumeral joint imbalance in female overhead activity athletes. J Strength Cond Res. 2008;22(1):140-145. doi:10.1519/JSC.0b013e31815f5634.

13. Outdoor Industry Foundation. 2005 Outdoor Recreation Participation Study. Boulder, CO: Outdoor Industry Foundation; 2006.
14. Pegoli L, Bain G, Badia A, Luchetti R, Beppu M. Hand injury in rock climbing. International Federation of Societies for Surgery of the Hand. 2015;5(3):16-20.

15. Rooks MD. Rock climbing injuries. Sports Med. 1997;4:261270. doi:10.2165/00007256-199723040-00005.

16. Sallis RE, Jones K, Sunshine S, Smith G, Simon L. Comparing sports injuries in men and women. Int J Sports Med. 2001;22(6):420-423. doi:10.1055/s-2001-16246.

17. Shea KG, Shea OF, Meals RA. Manual demands and consequences of rock climbing. J Hand Surg Am. 1992;17(2): 200-205. doi:10.1016/0363-5023(92)90390-B.

18. Warme WJ, Brooks D. The effect of circumferential taping on flexor tendon pulley failure in rock climbers. Am J Sports Med. 2000;28(5):674-678. 